\section{Case Reports in Oncology}

\title{
Achievement of Cure with Gefitinib in Advanced Lung Adenocarcinoma Harboring an Activating EGFR Mutation: A Case Report
}

Taiji Kuwata Kazue Yoneda Kenichi Kobayashi Rintarou Oyama Hiroki Matumiya Shuichi Shinohara Masaru Takenaka Soichi Oka Yasuhiro Chikaishi Naoko Imanishi Koji Kuroda Fumihiro Tanaka

Second Department of Surgery, School of Medicine, University of Occupational and Environmental Health, Kitakyushu, Japan

\section{Keywords}

Lung cancer · EGFR mutation · EGFR TKI

\section{Abstract}

Tyrosine kinase inhibitors (TKIs) of the epidermal growth factor receptor (EGFR) may achieve long-term survival in selected cases with advanced non-small cell lung cancer harboring activating mutations in the EGFR gene, but a cured case has not been reported yet. Here, we present the first case of EGFR-mutated lung adenocarcinoma cured with an EGFR-TKI, as the 75-year-old Japanese man has achieved complete response with gefitinib treatment and has survived without tumor 10 years after termination of gefitinib treatment.

(C) 2016 The Author(s)

Published by S. Karger AG, Basel 


\section{Introduction}

For non-small cell lung cancer (NSCLC) harboring activating EGFR mutations, tyrosine kinase inhibitors (TKIs) such as gefitinib are remarkably effective [1], and no cured case of advanced NSCLC has been reported yet. Here, we now report the first case of EGFR-mutated lung adenocarcinoma cured with gefitinib.

\section{Case Report}

A 75-year-old Japanese man with clinical stage IIIB (T1aN3M0) lung adenocarcinoma originating from the right upper lobe was admitted. Computed tomography (CT) revealed enlargement of the right tracheobronchial nodes and right supraclavicular nodes, and N3 disease was pathologically confirmed on histological sections biopsied from the supraclavicular nodes. Two cycles of chemotherapy with cisplatin (CDDP) plus paclitaxel (PTX) were performed, which provided partial response with disappearance of the supraclavicular nodes on CT. As down-staging to stage IIIA (N2) was achieved, thoracotomy with right upper lobectomy and mediastinal nodal dissection, without supraclavicular nodal dissection, followed by 4 cycles of adjuvant chemotherapy with CDDP plus PTX was performed.

Six months after the operation, CT revealed a re-enlargement of the right supraclavicular nodes, and the patient was diagnosed with postoperative recurrence. As an activating EGFR mutation (exon 19 deletion) was detected in surgical specimens of the primary tumor, gefitinib treatment was initiated. After 3 months of treatment, CT revealed disappearance of supraclavicular nodes. Gefitinib treatment was terminated 18 months after initiation due to severe rash (grade 3). Complete response has continued throughout, during and even after termination of gefitinib treatment. The patient has received no additional anti-tumor treatment and is still alive without tumor 10 years after the termination of gefitinib treatment.

\section{Discussion}

We present the case of a patient with EGFR-mutated lung adenocarcinoma who achieved complete response with gefitinib treatment and has survived without tumor for 10 years after termination of gefitinib treatment, which can be referred to as 'cure'.

Platinum-based chemotherapy is the standard first-line treatment for advanced NSCLC but provides only modest clinical benefit with a median overall survival of only 8-12 months and a median progression-free survival of 5-6 months [2]. The introduction of EFGR-TKIs has improved the prognosis of EGFR-mutated NSCLC patients with a median overall survival of 20-30 months and a median progression-free survival of 10-14 months [3]. More importantly, the majority of patients who initially respond to epidermal growth factor receptor (EGFR)-TKIs develop acquired resistance, but some patients may survive longer with EGFRTKI treatment. In fact, a retrospective Japanese study reported that 28 (8.4\%) of 335 NSCLC patients treated with gefitinib survived for more than 5 years and the longest survival time was more than 10 years (3,867 days) [4]. Another retrospective study from the Dana-Farber Cancer Institute reported a 5-year survival rate of $14.6 \%$ with a median follow-up duration 
of 89.8 months (range, 60.1-91.6 months) [5]. In addition, Kempf et al. [6] reported the case of a patient with metastatic EGFR-mutated lung adenocarcinoma who survived 10 years. Nevertheless, these long-term survival cases, including a few 10 -year survivors, may not be judged as cases with 'cure' because these patients survive with tumor and/or on treatment.

In contrast, it may be reasonable to judge the present case to be cured, because the patient has survived without tumor for 10 years while no treatment has been prescribed. To the best of our knowledge, therefore, the present case is the first advanced EGFR-mutated NSCLC case who achieved 'cure' with an EGFR-TKI. The present case had an exon 19 deletion and had no extrathoracic or brain metastasis, which have been identified in previous clinical studies [4] as significant factors to predict a favorable prognosis, especially long-term survival, in EGFR-mutated NSCLC treated with EGFR-TKIs and may have contributed to the achievement of 'cure' in the present case.

In conclusion, EGFR-TKIs may provide a long-term survival in NSCLC harboring activating EGFR mutations, and may achieve 'cure' in very selected cases as presented in the report.

\section{Statement of Ethics}

Informed consent was obtained from the patient.

\section{Disclosure Statement}

The authors have no conflicts of interest to report.

\section{References}

1 Kazandjian D, Blumenthal GM, Yuan W, He K, Keegan P, Pazdur R: FDA approval of gefitinib for the treatment of patients with metastatic EGFR mutation-positive non-small cell lung cancer. Clin Cancer Res 2016;22:1307-1312.

2 Sciller JH, Harrington D, Belani CP, Langer C, Sandler A, Krook J, Zhu J; Eastern Cooperative Oncology Group: Comparison of four chemotherapy regimens for advanced non-small-cell lung cancer. N Engl J Med 2002;346:92-98.

-3 Lee CK, Brown C, Gralla RJ, Hirsh V, Thongprasert S, Tsai CM, Tan EH, Ho JC, Chu da T, Zaatar A, Osorio Sanchez JA, Vu VV, Au JS, Inoue A, Lee SM, Gebski V, Yang JC: Impact of EGFR inhibitors in non-small cell lung cancer on progression-free survival: a meta-analysis. J Natl Cancer Inst 2013;105:595-605.

-4 Lin JJ, Cardarella S, Lydon CA, Dahlberg SE, Jackman DM, Jänne PA, Johnson BE: Five-year survival in EGFR-mutant metastatic lung adenocarcinoma treated with EGFR-TKIs. J Thorac Oncol 2016;11:556565.

5 Nishino K, Imamura F, Morita S, Mori M, Komuta K, Kijima T, Namba Y, Kumagai T, Yamamoto S, Tachibana I, Nakazawa Y, Uchida J, Minami S, Takahashi R, Yano Y, Okuyama T, Kumanogoh A: A retrospective analysis of 335 Japanese lung cancer patients who respond to initial gefitinib treatment. Lung Cancer 2013;82:299-304.

-6 Kempf E, Planchard D, Le Chevalier T, Soria JC: 10-year long-term survival of a metastatic EGFRmutated nonsmall cell lung cancer patient. Eur Respir J 2015;46:280-282. 